OPEN ACCESS

Edited by:

Mohsin Khan

Temple University, United States

Reviewed by:

Wenbin Liang,

University of Ottawa, Canada

Ngonidzashe B. Madungwe,

Amgen, United States

${ }^{*}$ Correspondence:

Yongchang Sun

suny@bjmu.edu.cn

Specialty section:

This article was submitted to

Redox Physiology,

a section of the journal

Frontiers in Physiology

Received: 27 September 2021

Accepted: 06 December 2021

Published: 24 December 2021

Citation:

Rao Y, Gai X, Xiong J, Le Y and Sun Y (2021) Transient Receptor Potential Cation Channel Subfamily V Member 4 Mediates Pyroptosis

in Chronic Obstructive Pulmonary

Disease. Front. Physiol. 12:783891.

doi: 10.3389/fphys.2021.783891

\section{Transient Receptor Potential Cation Channel Subfamily V Member 4 Mediates Pyroptosis in Chronic Obstructive Pulmonary Disease}

\author{
Yafei Rao, Xiaoyan Gai, Jing Xiong, Yanqing Le and Yongchang Sun* \\ Department of Respiratory and Critical Care Medicine, Peking University Third Hospital, Beijing, China
}

TRPV4, a calcium permeable cation selective channel, was found to be involved in chronic obstructive pulmonary disease (COPD) through releasing ATP and IL-1 $\beta$. Pyroptosis, a newly discovered pro-inflammatory cell death, was induced by cigarette smoke (CS) in airway epithelial cells (AECs). More recent studies indicated that blocking $\mathrm{Ca}^{2+}$ influx effectively inhibited pyroptosis. Therefore, we asked whether TRPV4 mediated CS-induced pyroptosis of AECs and hence participated in the pathogenesis of COPD. We found that pyroptosis and TRPV4 were upregulated in AECs from patients with COPD and long-term CS-exposed mice. Moreover, pharmacological inhibition or knockdown of TRPV4 function alleviated CS extract (CSE)-induced pyroptosis by inhibiting NACHT, LRP, PYD domains-containing protein 3 (NLRP3) inflammasome/activated caspase-1/gasdermin D pathway, decreasing the number of PI positive cells and lactate dehydrogenase $(\mathrm{LDH})$ release, decreasing the expression of pro- inflammatory interleukin gene (IL)-1 $\beta, \mathrm{IL}-8$, and $\mathrm{IL}-18$ expression, as well as increasing anti-inflammatory gene expression $[\mathrm{NAD}(\mathrm{P}) \mathrm{H}$ quinone dehydrogenase 1 (NQO1), superoxide dismutase 2 (mitochondrial) (MNSOD), and catalase, (CAT)]. Moreover, pharmacological inhibition or knockdown of TRPV4 function significantly relieved CSE-induced mitochondrial damage including decreased mitochondrial membrane potential, mitochondrial fusion protein (OPA1, MFN2) expression, and increased mitochondrial fission protein (DRP1, MFF) expression. Taken together, these findings indicate that TRPV4 mediates AEC pyroptosis via NLRP3/caspase-1/GSDMD pathway in COPD.

Keywords: chronic obstructive pulmonary disease (COPD), cigarette smoke, TRPV4, pyroptosis, inflammation, mitochondrial dysfunction

\section{INTRODUCTION}

Chronic obstructive pulmonary disease (COPD) is featured by chronic airway inflammation and pulmonary parenchymal destruction (Singh et al., 2019). Airway epithelial cells (AECs) form the first barrier of the respiratory system against irritants from the environment and play a critical role in the development or progression of COPD (Brusselle et al., 2011; Tuder and Petrache, 2012; Barnes, 2013).

Pyroptosis, a form of lytic cell death resulting from pathogenic infection or endogenous challenge, drives inflammation through inflammatory cytokines and danger molecules 
(Jorgensen and Miao, 2015; Broz and Dixit, 2016; Rathinam et al., 2019). Pyroptosis has been shown to be increased in a variety of diseases, such as sepsis, acute pancreatitis, renal ischemia reperfusion injury, diabetic cardiomyopathy and Alzheimer's disease (Man et al., 2017; Manthiram et al., 2017; Swanson et al., 2019; Feng et al., 2020; Xiao et al., 2020; Xie et al., 2020; Gao et al., 2021). Emerging evidence indicates that cigarette smoke (CS) exposure can induce pyroptosis of AECs, suggesting a critical role of pyroptosis in the pathogenesis of COPD (Zhang et al., 2021).

Transient receptor potential cation channel subfamily $\mathrm{V}$ member 4 (TRPV4) belongs to the transient receptor potential (TRP) family, comprising of calcium permeable cation selective channels (Clapham, 2003; Negri et al., 2019). Accumulating evidence indicates that TRPV4 is involved in a variety of lung diseases, including cough (Bonvini et al., 2016; Bonvini and Belvisi, 2017), asthma (Yao et al., 2019), COPD (Baxter et al., 2014), idiopathic pulmonary fibrosis (IPF) (Riteau et al., 2010), and acute respiratory distress syndrome (Balakrishna et al., 2014). Interestingly, a previous study suggested that TRPV4 participated in the pathogenesis of COPD, by observing that TRPV4 mRNA was upregulated in lung tissues, alveolar macrophages and bronchial epithelial cells from COPD patients. Additionally, cigarette smoke extract (CSE)-induced ATP and IL- $1 \beta$ release was mediated by TRPV4 (Baxter et al., 2014). But the exact role of TRPV4 in the pathogenesis of COPD still awaits investigation. More recently, another study indicated that blocking $\mathrm{Ca}^{2+}$ influx effectively inhibited pyroptosis (Wang et al., 2020). Therefore, we asked whether TRPV4 played a role in the pathogenesis of COPD by mediating pyroptosis of AECs.

In this study, we evaluated pyroptosis and TRPV4 expression in AECs from patients with COPD and long-term CS-exposed mice. We found that pyroptosis and TRPV4 were significantly upregulated in AECs from COPD patients and long-term CS-exposed mice. Moreover, pharmacological inhibition or knockdown of TRPV4 function attenuated protein expressions of NLRP3 inflammasome, activated caspase-1 and gasdermin $\mathrm{D}-\mathrm{N}$ (GSDMD-N), decreased the number of PI positive cells and lactate dehydrogenase $(\mathrm{LDH})$ release, decreased the expressions of pro- inflammatory interleukin (IL)-1 $\beta$, IL-8, and IL-18, while increased anti-inflammatory gene expressions including $[\mathrm{NAD}(\mathrm{P}) \mathrm{H}$ quinone dehydrogenase 1 (NQO1), superoxide dismutase 2 (mitochondrial) (MNSOD) and catalase $(C A T)]$. Furthermore, pharmacological inhibition or knockdown of TRPV4 function significantly relieved CSEinduced mitochondrial damage, as manifested by increased mitochondrial membrane potential, mitochondrial fusion protein (OPA1, MFN2) expression and decreased mitochondrial fission protein (DRP1, MFF) expression.

\section{MATERIALS AND METHODS}

\section{Human Subjects}

The human study was approved by the Ethics Review Committee of Peking University Third Hospital. All study subjects signed an informed consent form. Totally, 24 subjects were recruited and subdivided into a healthy non-smoking (HNS) group, a smoker group and a COPD group. HNS controls were defined as neversmokers having a post-bronchodilator $\mathrm{FEV}_{1} / \mathrm{FVC} \geq 0.7$. Smokers were defined as having a smoking history of $\geq 10$ pack-years with a post-bronchodilator $\mathrm{FEV}_{1} / \mathrm{FVC} \geq 0.7$. COPD patients were defined as having $\geq 10$ pack-years of smoking history, and a postbronchodilator $\mathrm{FEV}_{1} / \mathrm{FVC}<0.7$. All COPD patients recruited were clinically stable. The study subjects had received lung surgery for solitary tumors, and lung tissues with a maximum distance from the tumor were collected by a pathologist.

\section{The Mouse Model of Chronic Obstructive Pulmonary Disease}

All the procedures and protocols carried out in the mouse experiments were approved by Animal Care Committee of Peking University Third Hospital. 6-8week old C57BL/6 female mice, weight 22-25 g, were provided by Beijing Vital River Experimental Animal Company (Beijing, China). All the mice were housed in a specific pathogen-free facility with free access to sterilized food and water. These mice were exposed to filtered air or CS (Baisha cigarettes with filter, Hunan, China) with a nose-only smoke exposure system (SG-300; SIBATA, Saitama, Japan), which has been described in our previous study (Zhou et al., 2019; Xiong et al., 2020). Each mouse was exposed to cigarettes or filtered air for two times a day, 50 min each time with 20-min smoke -free intervals, 5 days a week, for 24 weeks consecutively.

\section{Lung Histology and Measurement of Emphysema}

Mouse lung tissues were obtained, fixed with $4 \%$ paraformaldehyde for $24 \mathrm{~h}$, embedded with paraffin, then cut into sections of $4 \mu \mathrm{m}$ thickness, followed by H\&E staining. The airspace enlargement in mice was quantified by the mean linear intercept (MLI) and destruction of alveolar walls in mice was quantified by destructive index (DI), which has been described in our previous studies (Zhou et al., 2019; Xiong et al., 2020).

\section{Immunohistochemistry of Lung Tissues}

Lung tissues were cut into $5 \mu \mathrm{m}$ sections, incubated in $0.3 \%$ $\mathrm{H}_{2} \mathrm{O}_{2}-\mathrm{CH}_{3} \mathrm{OH}$ for $15 \mathrm{~min}$ for blocking endogenous peroxidase activity, then treated with citrate buffer ( $\mathrm{pH}$ 6.0) using a microwave oven for $15 \mathrm{~min}$ to retrieve antigen, and followed by blocking with $5 \%$ bovine serum albumin for $30 \mathrm{~min}$ at room temperature (RT). Subsequently, the tissues were incubated overnight with antibodies against TRPV4 (1:100, Alomone labs, Jerusalem, Israel), human gasdermin D (GSDMD) (1:200, Abcam, Cambridge, United Kingdom), mouse GSDMD (1:1,500, Bioss Biotechnology Co., Ltd, Beijing, China), human gasdermin D N-terminal fragment (GSDMD-N) (1:400, Abcam), and mouse gasdermin D C-terminal fragment (GSDMD-C) (1:400, Abcam). The tissues were then incubated with horseradish peroxidase (HRP)-conjugated goat anti-rabbit IgG (ZSGB-Bio, Beijing, China) at $37^{\circ} \mathrm{C}$ for $30 \mathrm{~min}$. Finally, slides were visualized by staining with a 3,3'-Diaminobenzidine (DAB) detection system kit (ZSGB-Bio). Images were photographed by a microscope and 
analyzed by Image-Pro Plus 6.0 software (Media Cybernetics, MD, United States).

\section{Cell Culture and Antagonist Treatment}

Human bronchial epithelial cells (16HBEs) were purchased from Bai Ye Biotechnology Center (Shanghai, China). Cells were cultured in Roswell Park Memorial Institute medium supplemented with $10 \%$ fetal calf serum (Gibco, Grand Island, NY, United States), 1\% penicillin/streptomycin (Gibco, Grand Island, $\mathrm{NY}$, United States) at $37^{\circ} \mathrm{C}$ with $5 \% \mathrm{CO}_{2} .16 \mathrm{HBEs}$ were pretreated with $10 \mu \mathrm{M}$ of a TRPV4 antagonist [GSK205 (catalog number HY120691A, MCE, Monmouth Junction, NJ, United States)] for $1 \mathrm{~h}$ and then stimulated with medium only or with CSE for another $24 \mathrm{~h}$.

\section{Short Interfering RNA Knockdown of Transient Receptor Potential Cation Channel Subfamily V Member 4}

For transient transfection, cells were cultured in six-well culture plates overnight prior to transfection. Short interfering (si) RNA or control oligonucleotide (GenePharma, Jiang Su, China) were incubated with $4 \mu \mathrm{l}$ of JetPRIME ${ }^{\circledR}$ in $200 \mu \mathrm{l}$ of jetPRIME buffer (Polyplus-transfection) for $15 \mathrm{~min}$ at room temperature. A final concentration of $50 \mathrm{nM}$ of TRPV4 siRNA or control oligonucleotide were added to cell culture plates. After $24 \mathrm{~h}$, CSE was added and stimulated for another $24 \mathrm{~h}$. Finally, cells were harvested for subsequent experiments.

\section{Cigarette Smoke Extract Preparation}

CSE was prepared according to our previous study (Zhou et al., 2019). Briefly, five cigarettes (Baisha, China Tobacco Industry Co., Ltd., Hunan, China) were bubbled through $10 \mathrm{ml}$ medium at a constant velocity (Hyclone, Logan, UT, United States). The solution was filtered through a $0.22 \mu \mathrm{m}$ filter, which was served as the $100 \%$ CSE work solution.

\section{Cell Viability Assessment}

Cell viability was detected by the cell counting Kit-8 (CCK8) assay kit (KeyGEN Biotechnology Co., Ltd., Jiangsu, China). In brief, Cells were treated with medium or different concentrations of CSE or GSK205 for $24 \mathrm{~h}$, then washed with PBS for three times and incubated with CCK8 reagent for another $2 \mathrm{~h}$. Subsequently, the absorbance was assessed by spectrophotometer at $450 \mathrm{~nm}$.

\section{Western Blotting Analysis}

To assess the protein expression levels of TRPV4, NLRP3, Caspase-1, GSDMD, GSDMD-N, GSDMD-C, dynamin-related protein 1 (DRP1), mitochondrial fission factor (MFF), OPA1 mitochondrial dynamin like GTPase (OPA1), and mitofusin 2 (MFN2), lung tissue or 16HBE cells were lysed and equal amount of protein was subjected to 8 or $10 \%$ SDS-PAGE, subsequently, protein was transferred to $0.22 \mu \mathrm{m}$ PVDF membranes (MerckMillipore, Carrigtwohill, Ireland). The membranes were blocked with 5\% non-fat milk powder and then incubated with antibodies overnight at $4^{\circ} \mathrm{C}$ : GAPDH, $\beta$-actin, DRP1, MFF, OPA1, MFN2 (1:1,000, Cell Signaling Technology, Danvers,
TABLE 1 | Clinical characteristics of the study subjects.

\begin{tabular}{lccc}
\hline & HNS & Smoker & COPD \\
\hline Subjects (all male) & 8 & 8 & 8 \\
Age (years) & $61.43 \pm 15.96$ & $64.75 \pm 7.96$ & $68.13 \pm 8.45$ \\
$\begin{array}{l}\text { FEV1/FVC (\%), } \\
\text { post-bronchodilator }\end{array}$ & $79.14 \pm 3.76$ & $76.09 \pm 4.09$ & $63.82 \pm 3.8^{*}$ \\
$\begin{array}{l}\text { FEV\%pred, } \\
\text { post-bronchodilator }\end{array}$ & $98.86 \pm 22.45$ & $86.16 \pm 13.16$ & $65.61 \pm 11.35^{*}$ \\
$\begin{array}{l}\text { Smoking index } \\
\text { (pack-years) }\end{array}$ & 0 & $41.38 \pm 23.01^{\#}$ & $55 \pm 21.38^{\star}$ \\
\hline
\end{tabular}

Smoking index $=$ average number of cigarettes per day (pack) $\times$ number of years of smoking history (years); FEV1, forced expiratory volume in $1 \mathrm{~s}$; FVC, force vital capacity; HNS, healthy none smoker; COPD, chronic obstructive pulmonary disease.

\#There was significant difference between Smokers and the HNS.

*There was significant difference between Smokers and COPD patients.

MA, United States), Caspase-1, NLRP3 (1:1,000, RD system, Minneapolis, MN, United States), GSDMD, GSDMD-N, and GSDMD-C (1:1,000, Abcam, Cambridge, United Kingdom).

Membranes were then washed and incubated with HRPconjugated donkey anti-mouse IgG or antirabbit IgG antibody for $1 \mathrm{~h}$ at RT. Finally, membranes were visualized with enhanced chemiluminescence (MerckMillipore, Carrigtwohill, Ireland). Quantitative images were analyzed using Image J software.

\section{Quantitative Real-Time Reverse Transcription PCR}

Gene expressions of TRPV4, IL1B, IL8, IL18, NQO1 [NAD(P)H quinone dehydrogenase 1], MNSOD [superoxide dismutase 2 (mitochondrial), and CAT (catalase)] were determined by qRT-PCR. RNA was isolated by TRIzol reagent (Thermo Fisher Scientific, MA, United States) and the concentration was determined by Nano Drop 2000. Then RNA was reverse transcribed into cDNA using HiScript III RT Supermix for $\mathrm{qPCR}(+\mathrm{gDNA}$ wiper) Kit (Vazyme, Nanjing, China). The $\mathrm{qPCR}$ reactions were performed on the Applied Biosystems $^{\circledR}$ QuantStudio $^{\circledR} 5$ in a $20 \mu$ l reaction system by using ChamQ Universal SYBR qPCR Master Mix Kit (Vazyme, Nanjing, China). The primer sequences were: human TRPV4: $5^{\prime}$-GGCTTTTCCTCTCTCCTCCC-3' (forward) and 5'-AGGGTGGACTCCAGCAGAT-3' (reverse); mouse TRPV4: $5^{\prime}$-TCCTGAGGCCGAGAAGTACA-3' (forward) and 5'-TCCC CCTCAAACAGATTGGC-3' (reverse); human GAPDH/Gapdh: 5'-AAATCAAGTGGGGCGATGCTG-3' (forward) and 5'-GCA GAGATGATGACCCTTTTG-3'(reverse); mouse GAPDH/ Gapdh: 5'-AAATGGTGAAGGTCGGTGTGAAC-3' (forward) and $5^{\prime}$-CAACAATCTCCACTTTGCCACTG-3'(reverse); $I L-1 \beta$ : $5^{\prime}$-ACTGAGAGTGATTGAGAGTGGAC-3' (forward) and 5'-AACCCTCTGCACCCAGTTTTC-3' (reverse); IL-8: 5'-AT GATGGCTTATTACAGTGGCAA-3' (forward) 5'-GTCGGAGA TTCGTAGCTGGA-3' (reverse); IL-18: $5^{\prime}$-TCTTCATTGACC AAGGAAATCGG-3' (forward) and 5'-TCCGGGGTGCATTAT CTCTAC-3' (reverse); NQO1: 5'-CAGTGGCATGCACCCA GGGAA-3' (forward) and 5'-GCATGCCCCTTTTAGCCTTG 


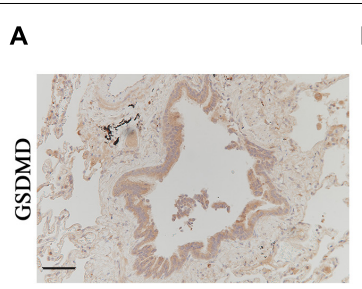

B
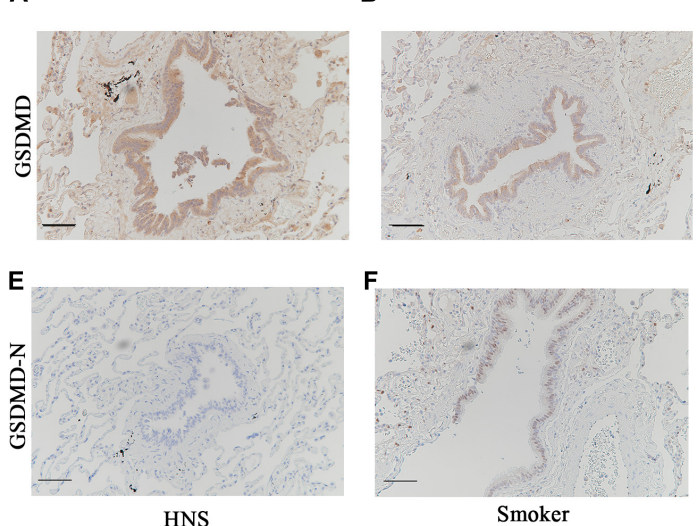

Smoker

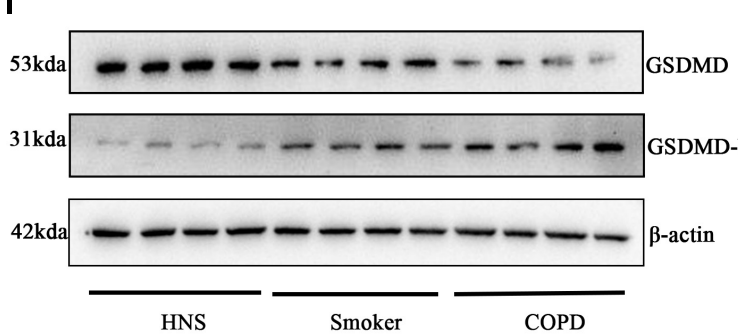

C

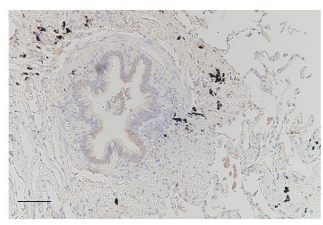

G

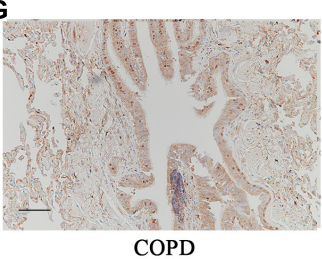

D

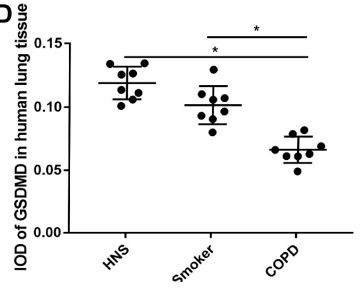

$\mathrm{H}$ 웅
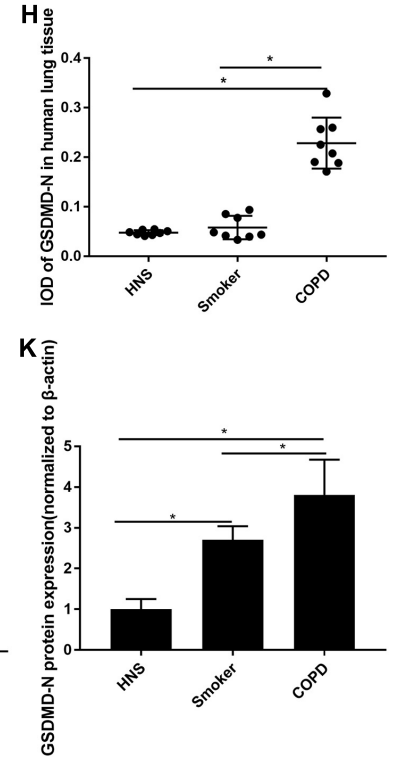

FIGURE 1 | GSDMD-N expression was upregulated in AECs from patients with COPD. (A-C) Representative immunohistochemical staining of GSDMD in AECs of HNS, Smoker and COPD groups. (E-G) Representative immunohistochemical staining of GSDMD-N in AECs of HNS, Smoker and patients with COPD. (D-H) The integrated optical density (IOD) in immunohistochemistry of GSDMD and GSDMD-N. Bar: $100 \mu \mathrm{m} . N=8$ per group. ${ }^{*} P<0.05$. (I-K) GSDMD, GSDMD-N protein expression in lung tissue homogenates from HNS, Smoker and patients with COPD were detected by Western blotting. $N=8$ per group. ${ }^{\star} P<0.05$.

GCA-3' (reverse); MNSOD: 5'-ACAGGCCTTATTCCACTGCT$3^{\prime}$ (forward) and 5'-CAGCATAACGATCGTGGTTT-3' (reverse); and CAT: 5'-TAAGACTGACCAGGGCATC-3' (forward) and $5^{\prime}$-CAAACCTTGGTGAGATCGAA- $3^{\prime}$ (reverse). The real-time PCR conditions were: $95^{\circ} \mathrm{C}$ for $15 \mathrm{~min}$, followed by 40 cycles of $95^{\circ} \mathrm{C}$ for $10 \mathrm{~s}$ and $60^{\circ} \mathrm{C}$ for $32 \mathrm{~s}$. Relative gene expression to control was calculated with the $2^{-\Delta} \Delta$ $C T$ method. Gene expression was calculated relative to that of GAPDH/Gapdh in triplicates.

\section{$\mathrm{Ca}^{2+}$ Influx Measurement}

Calbryte 520 AM (AAT Bioquest, San Francisco, CA, United States) was used to assess the intracellular $\mathrm{Ca}^{2+}$ levels. In brief, cells were loaded with $2 \mathrm{mM}$ Calbryte $520 \mathrm{AM}$ at $37^{\circ} \mathrm{C}$ in the dark for $1 \mathrm{~h}$, and then washed with PBS. Thereafter, cells were stimulated with CSE for $20 \mathrm{~min}$. The intracellular $\mathrm{Ca}^{2+}$ influx was recorded by a confocal laser scanning microscope for 20 consecutive minutes, at $5 \mathrm{~s}$ per frame.

\section{Measurement of Intracellular Reactive Oxygen Species}

$2^{\prime}, 7^{\prime}$-dichlorofluorescin diacetate (DCFH-DA) (Beyotime Biotechnology Co., Ltd., Shanghai, China) was used to assess the intracellular ROS with fluorescent microscopy. In brief, cells were pretreated with GSK205 $10 \mu \mathrm{M}$ for $1 \mathrm{~h}$, and then stimulated with medium only or CSE for another $24 \mathrm{~h}$. These cells then underwent incubation with $10 \mu \mathrm{M}$ DCFH-DA for $20 \mathrm{~min}$ at $37^{\circ} \mathrm{C}$. Finally, the intracellular ROS was assessed at 488/525 nm using fluorescent microscopy (Leica, Germany) and analyzed by Image-Pro Plus 6.0 software (Media Cybernetics, MD, United States).

\section{Measurement of Mitochondrial Reactive Oxygen Species}

Mitochondrial ROS was assessed by MioSox Red (Invitrogen, Life Technologies, Carlsbad, CA, United States). Briefly, after corresponding treatment, cells were stained with $5 \mu \mathrm{M}$ Mio Sox Red in the dark for $10 \mathrm{~min}$. The mitochondrial ROS was determined at 510/580 $\mathrm{nm}$ using fluorescent microscopy (Leica, Germany) and analyzed by Image-Pro Plus 6.0 software (Media Cybernetics, MD, United States).

\section{Lactate Dehydrogenase Assay}

$\mathrm{LDH}$ was assessed using an LDH assay kit (KeyGEN Biotechnology Co., Ltd). Briefly, cells were handled as described above, then the supernatants were harvested and assessed for 

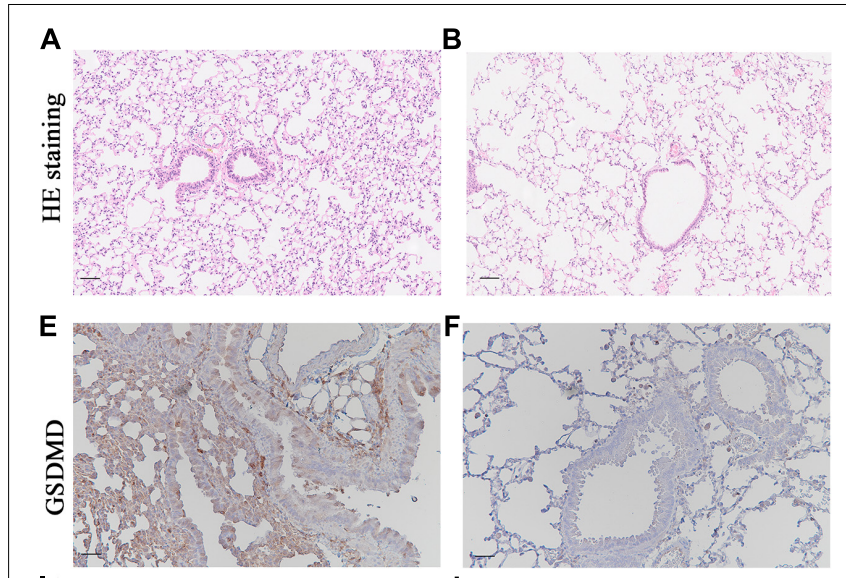

I

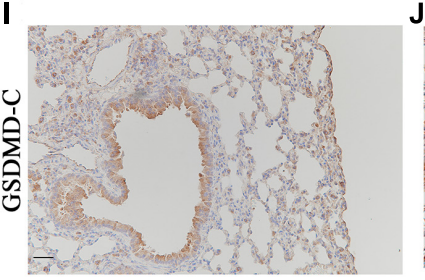

K

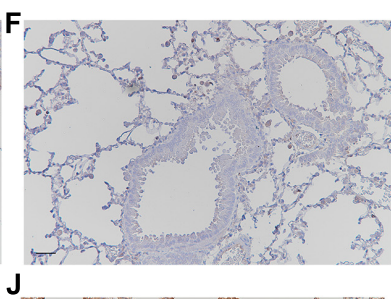

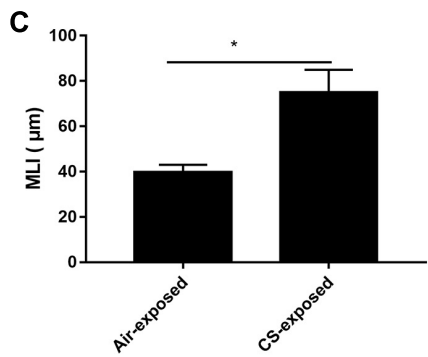
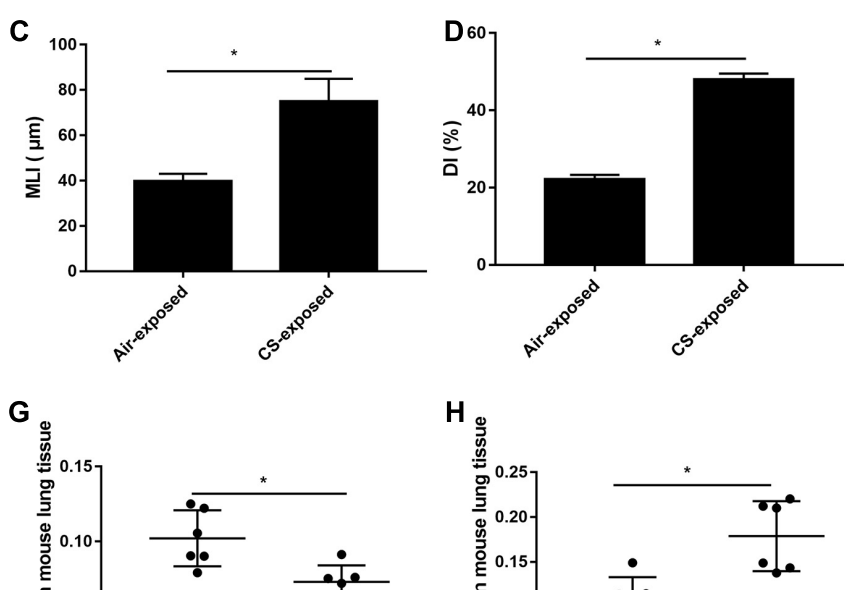

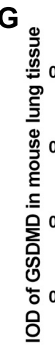
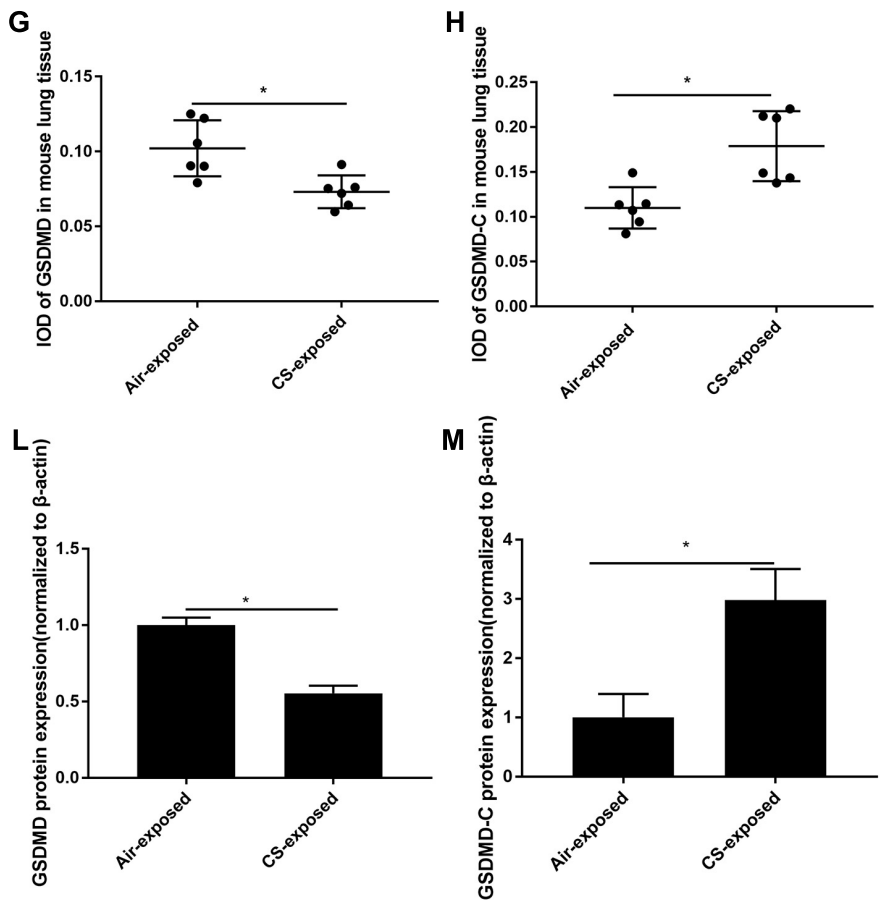

FIGURE 2 | GSDMD-C expression was upregulated in AECs from mouse COPD. (A,B) H\&E-stained lung sections Bar: 50 m. (C,D) Mean linear intercept (MLI) and destructive index (DI) was measured. (E,F) Representative immunohistochemical staining of GSDMD in AECs of CS-exposed group and air-exposed group. (I,J) Representative immunohistochemical staining of GSDMD-C in AECs of air-exposed and CS-exposed mice. (G,H) The integrated optical density (IOD) in immunohistochemistry of GSDMD and GSDMD-C. Bar: $50 \mu \mathrm{m} . N=6$ per group. ${ }^{\star} P<0.05$. (K-M) GSDMD,GSDMD-C protein expression in lung tissue homogenates from air-exposed mice $(N=4)$ and CS-exposed mice $(N=6)$ were detected by Western blotting. ${ }^{\star} P<0.05$.

$\mathrm{LDH}$ release with a microplate reader at $490 \mathrm{~nm}$ according to the manufacturer's instruction.

\section{Hoechst 33342/PI Staining}

Hoechst 33342/PI staining was measured using apoptosis and necrosis Assay Kit (Beyotime Biotechnology Co., Ltd., Shanghai, China). After corresponding treatments described above, cells were stained with $5 \mu$ l Hoechst 33342 and $5 \mu \mathrm{l}$ PI in $1 \mathrm{ml}$ staining buffer solution on ice in the dark for $20 \mathrm{~min}$. Then, PI positive cells were photographed by confocal microscopy.

\section{Measurement of Mitochondrial Membrane Potential}

MMP was assessed with Mitotracker deep red (Invitrogen, Life Technologies). After corresponding treatment, cells were incubated with $50 \mathrm{nM}$ Mitotracker deep red for $30 \mathrm{~min}$ at $37^{\circ} \mathrm{C}$, and then detected at $644 / 655 \mathrm{~nm}$ by flow cytometry.

\section{Mitochondrial Morphology Detection}

After corresponding treatment, mitochondria were labeled with the MitoTracker Green (Beyotime Biotechnology Co., Ltd., Shanghai, China) for $30 \mathrm{~min}$ in the dark. The mitochondrial morphology was photographed by a confocal microscope (Leica SP5, Germany). MitoTracker Green fluorescence intensity, mitochondrial Aspect Ratio and Form Factor was analyzed by Image J software. A minimum of 20 mitochondria were analyzed.

After corresponding treatment, cells were collected and washed with PBS for subsequent transmission electron microscopic (TEM) detection. Firstly, cells were added with Glutaraldehyde $(2.5 \%)$ carefully and fixed at $4^{\circ} \mathrm{C}$ for $1.5 \mathrm{~h}$. Then, the mixture was washed three times with sucrose buffer, 
fixed, dehydrated, soaked, and embedded, ultrathin sectioned and heavy metal stained. The mitochondrial morphology was assessed by JEM1400 PLUS. Mitochondrial Aspect Ratio and Form Factor in mitochondrial were calculated using Image J software. A minimum of 30 mitochondria were analyzed.

\section{Statistical Analysis}

All data were shown as mean \pm standard deviation (SD). Statistical analysis was processed with GraphPad Prism 7. Students' $t$-tests or one-way ANOVA with Bonferroni post hoc test (equal variances assumed) or Dunnett's T3 post hoc test (equal variances not assumed) were used when applicable. $P$-values less than 0.05 were considered statistically significant.

\section{RESULTS}

\section{Clinical Characteristics of Human Subjects}

Eight COPD patients, 8 smokers, and 8 HNS controls were enrolled. Table 1 shows the clinical data of the subjects.

\section{Increased Pyroptosis of Airway Epithelial Cells in Human and Mouse Chronic Obstructive Pulmonary Disease}

A previous in vitro study has implicated pyroptosis in the pathogenesis of COPD (Zhang et al., 2021), however, the expression of pyroptosis in human and mouse COPD were still unknown. Therefore, we investigated the status of pyroptosis of AECs in patients with COPD, smokers and HNS, by performing immunohistochemical staining of lung sections. Patients with COPD showed reduced protein level of GSDMD (Figures 1AD), but increased protein level of GSDMD-N (Figures 1E-H) in AECs, as compared to smokers and HNS. Moreover, Western blotting analysis revealed decreased GSDMD but increased GSDMD-N protein levels in lung tissue homogenates from COPD patients as compared to smokers and HNS (Figures 1I$\mathbf{K})$, indicating enhanced pyroptosis in patients with COPD.

We also performed immunohistochemistry for the detection of pyroptosis of AECs in lung sections from mice with CSinduced COPD. Mice in the 6-month CS-exposed group showed enlargement of airway spaces (Figures 2A,B), with significant increase in MLI and DI (Figures 2C,D), which is in line with typical changes of COPD. Consistent with the observations in human subjects, CS-exposed mice showed reduced protein level of GSDMD (Figures 2E-G), but increased protein level of GSDMD-C (a pyroptosis marker in mice) in AECs, as compared to air-exposed mice (Figures $2 \mathbf{H}-\mathbf{J}$ ). Control antibody staining was illustrated in Supplementary Figures 1, 2. Western blotting analysis also demonstrated reduced GSDMD but increased GSDMD-C protein levels in lung tissue homogenates from CSexposed mice as compared to air-exposed mice (Figures $\mathbf{2} \mathbf{K}-\mathbf{M}$ ). Collectively, these data demonstrated that there was a significant increase in pyroptosis of AECs in human and mouse COPD.

\section{Transient Receptor Potential Cation Channel Subfamily V Member 4 Expression Was Upregulated in Airway Epithelial Cells From Human and Mouse Chronic Obstructive Pulmonary Disease}

A previous study observed increased TRPV4 mRNA in lung homogenates from patients with COPD in relative to HNS and smokers (Baxter et al., 2014). To further evaluate the protein expression of TRPV4 in COPD, we carried out immunohistochemistry on lung sections from COPD patients. Immunohistochemistry analysis detected increased TRPV4 protein expression in AECs from COPD patients and smokers compared to HNS (Figures 3A-C,F). Similarly, TRPV4 expression was upregulated in AECs from CS-exposed mice as compared to the air-exposed ones (Figures 3D,E,G). CS-exposed mice also showed higher TRPV4 mRNA levels in lung tissues, as compared to the air-exposed controls (Figure 3H). Furthermore, Western blotting analysis observed increased TRPV4 protein level in lung homogenates from CS-exposed mice (Figures 3I,J). Taken together, these results demonstrated that TRPV4 was upregulated in AECs from COPD patients and long-term CSexposed mice.

\section{Transient Receptor Potential Cation Channel Subfamily V Member Mediates CS Extract-Induced Pyroptosis via the $\mathrm{Ca}^{2+} / \mathrm{NACHT}$, LRP, PYD Domains-Containing Protein $3 /$ Caspase-1/Gasdermin D Axis}

Emerging evidence indicates that CS exposure can induce pyroptosis of AECs, suggesting a critical role of pyroptosis in the pathogenesis of COPD (Zhang et al., 2021), but the molecules mediating CS-induced pyroptosis was not clear. To determine whether TRPV4 mediates pyroptosis in CS-exposed 16HBEs, we performed pharmacological inhibition or knockdown of TRPV4 function in 16HBEs before stimulation with CSE. TRPV4 siRNA reduced TRPV4 mRNA level by $90 \%$ and protein level by $70 \%$ (Supplementary Figures 3A-C). To determine the optimal dose of CSE for in vitro experiments, we firstly assessed the cellular toxicity of CSE at concentrations ranging from 2 to $10 \%$. After stimulation with $4 \%$ CSE for $24 \mathrm{~h}$, about $80 \%$ cells survived (Supplementary Figure 4). Accordingly, 4\% CSE exposure for $24 \mathrm{~h}$ was applied in following experiments. In addition, TRPV4 inhibitor showed no cellular toxicity at a range from $100 \mathrm{nM}$ to $100 \mu \mathrm{M}$ (Supplementary Figure 5). After incubation with CSE for $24 \mathrm{~h}, 16 \mathrm{HBEs}$ were harvested for Western blotting and qRTPCR. We found that CSE induced significant increase in TRPV4 expression at the mRNA level and the protein level (Figures 4AC), indicating that CS promoted TRPV4 expression, which was consistent with our observations in humans and mice.

As a previous study indicated that blocking $\mathrm{Ca}^{2+}$ influx could effectively inhibit pyroptosis (Wang et al., 2020), we thus measured $\mathrm{Ca}^{2+}$ influx in 16HBEs after corresponding treatments. We noted a time-dependent $\mathrm{Ca}^{2+}$ influx in $16 \mathrm{HBEs}$ 


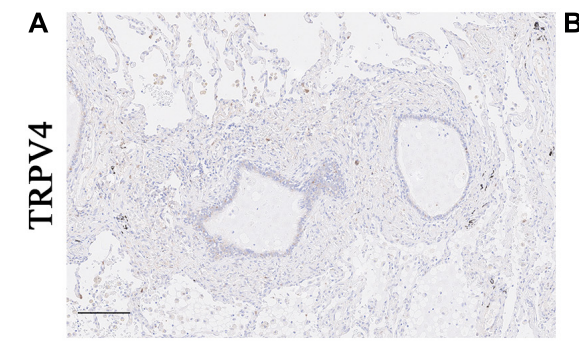

HNS

D

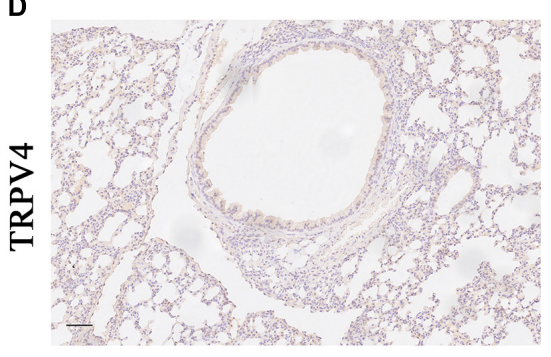

Air-exposed

G

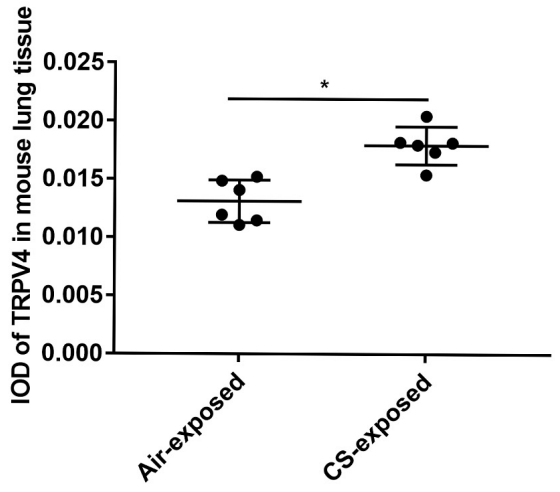

B

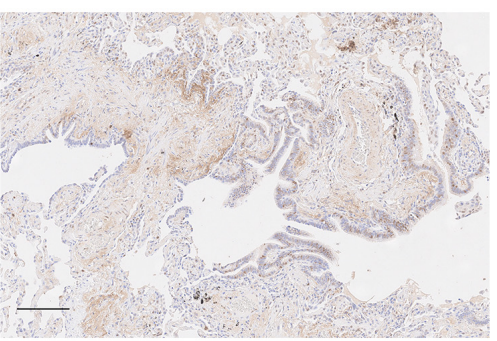

Smoker

E

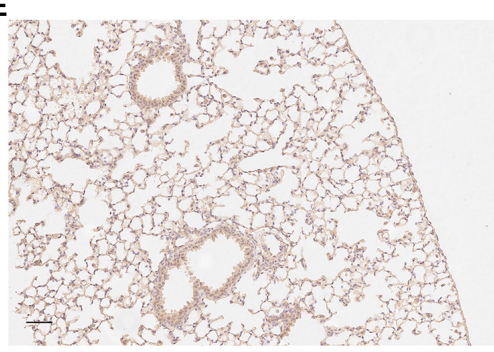

CS-exposed

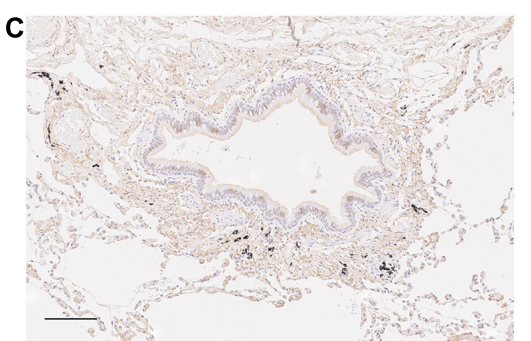

COPD

F

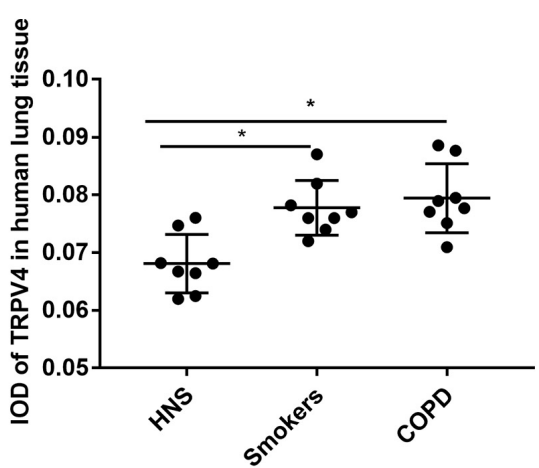

H

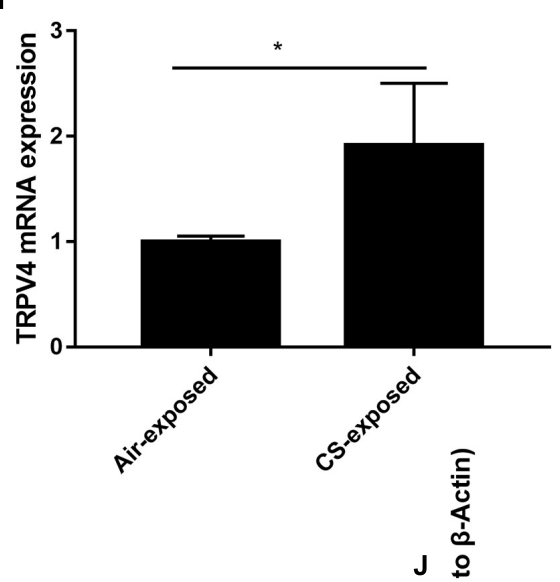

I

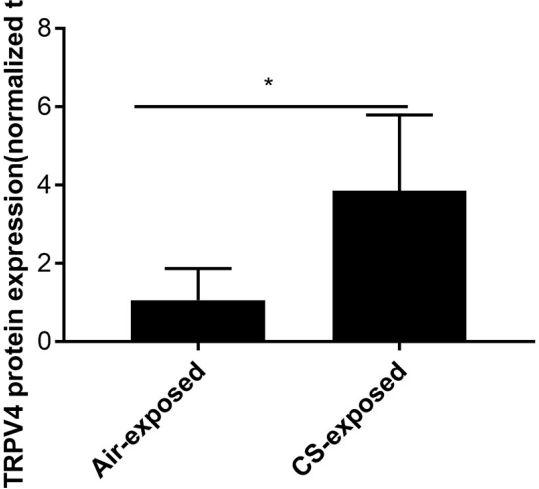

FIGURE 3 | TRPV4 expression was up-regulated in AECs in human and mouse COPD. (A-C) Representative immunohistochemical staining of TRPV4 in lung sections of HNS, Smoker and patients with COPD. (F) The integrated optical density (IOD) in immunohistochemistry of TRPV4. Bar: $100 \mu \mathrm{m}$. $N=8$ per group. ${ }^{\star} P<$ 0.05. (D,E) Representative immunohistochemical staining of TRPV4 in AECs of air-exposed mice and CS-exposed mice. (G) The integrated optical density (IOD) in immunohistochemistry of TRPV4. Bar: $50 \mu \mathrm{m} . N=6$ per group. ${ }^{\star} P<0.05$. (H) The lung homogenates of CS-exposed mice displayed increased TRPV4 mRNA expression compared with air-exposed mice. $(\mathbf{I}, \mathbf{J})$ The lung homogenates of CS-exposed mice $(N=5)$ demonstrated increased TRPV4 expression at protein level compared with air-exposed mice $(N=4) .{ }^{\star} P<0.05$. 
A

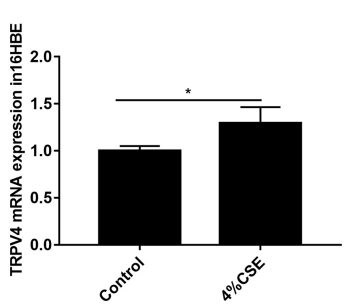

D

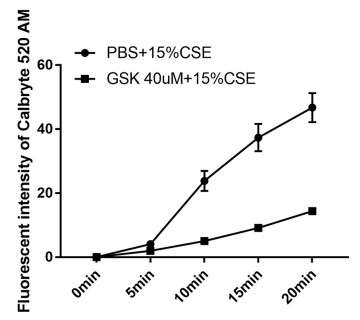

$\mathbf{F}$
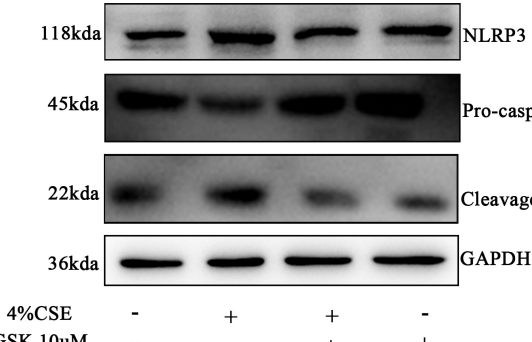

$\begin{array}{cccc}4 \% \mathrm{CSE} & - & + & + \\ \mathrm{GSK} 10 \mathrm{uM} & - & - & +\end{array}$

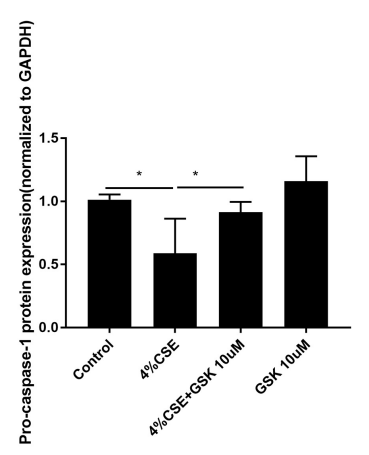

M

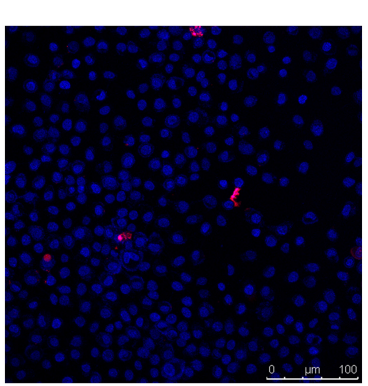

Control
B

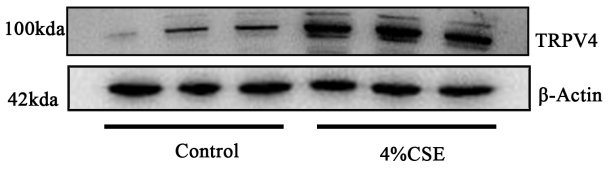

E

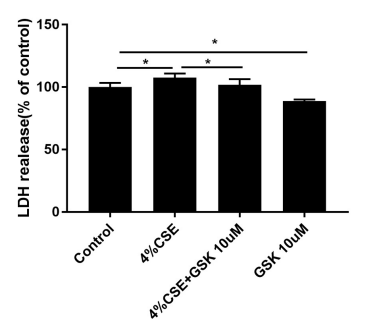

G

$53 \mathrm{kda}$

$53 \mathrm{kda}$

ro-caspase-1

Cleavage caspase- 1

GAPDH
C
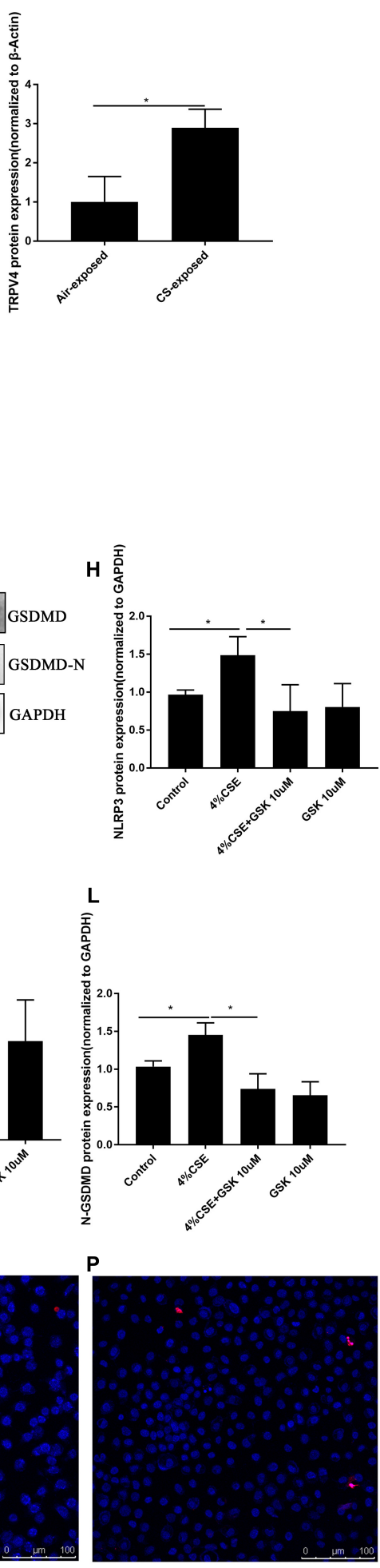

GSK $10 \mathrm{uM}$

FIGURE 4 | TRPV4 mediates CSE-induced pyroptosis via the $\mathrm{Ca}^{2+}$ /NLRP3/Caspase-1/GSDMD axis. 16HBEs were pretreated with GSK205 (10 $\left.\mu \mathrm{M}\right)$ for $1 \mathrm{~h}$, then stimulated with $4 \%$ CSE for another $24 \mathrm{~h}$. (A-C) CSE induced upregulated expression of TRPV4 at mRNA level and protein level. ${ }^{*} P<0.05 . N=3$ independent experiments. (D) Pharmacological inhibition with GSK 205 blocked $\mathrm{Ca}^{2+}$ influx induced by CSE. $N=10$ cells. (E) LDH release induced by CSE was abrogated by pharmacological inhibition of TRPV4 with GSK 205. ${ }^{\star} P<0.05 . N=6$ independent experiments. (F-L) Pharmacological inhibition with GSK 205 decreased the level of NLRP3, Pro-Caspase-1, Cleavage cspase-1, GSDMD, GSDMD-N induced by CSE. ${ }^{*} P<0.05 . N \geq 3$ independent experiments. (M-P) Pharmacological inhibition with GSK 205 abrogated PI positive cell induced by CSE. Bar: $100 \mu \mathrm{m}$. 

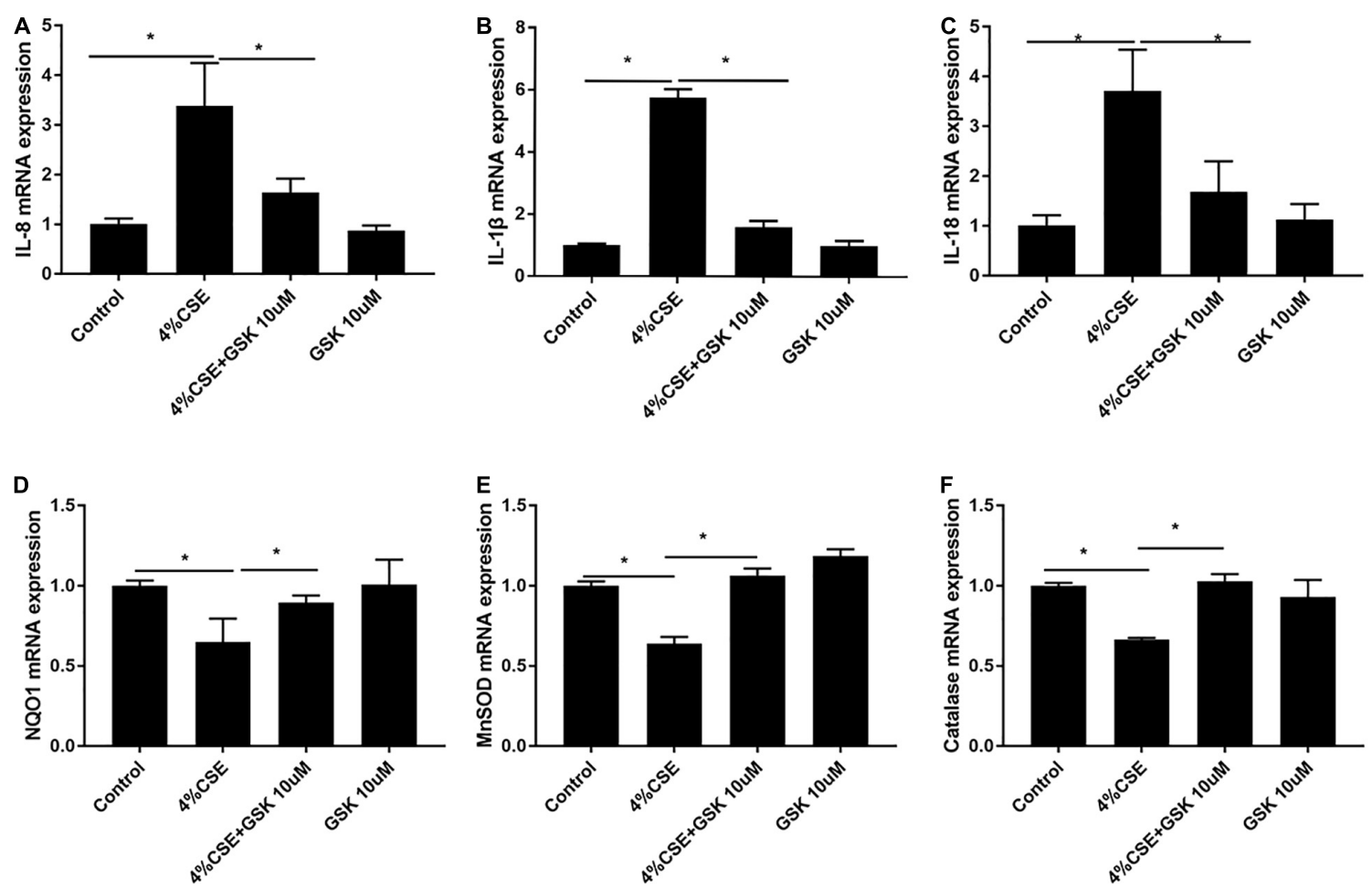

FIGURE 5 | Pharmacological inhibition of TRPV4 channel with GSK205 attenuated inflammatory genes while upregulated antioxidant gene expression. 16HBEs

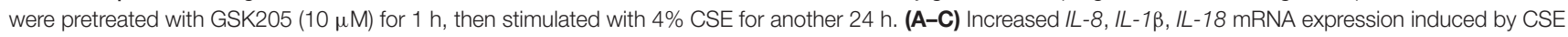
were abrogated by pharmacological inhibition with GSK205. (D-F) Decreased NQO1, MNSOD, and Catalase mRNA expression induced by CSE were improved by pharmacological inhibition with GSK205. ${ }^{*} P<0.05 . N=3$ independent experiments.

induced by CSE, while pharmacological inhibition with GSK205 significantly attenuated this $\mathrm{Ca}^{2+}$ influx (Figure 4D). To further confirm this observation, we carried out in vitro TRPV4 siRNA experiments, which demonstrated that TRPV4 gene knockdown significantly blocked $\mathrm{Ca}^{2+}$ influx induced by CSE (Supplementary Figure 3D). To further explore whether TRPV4 mediates pyroptosis in CS-exposed 16HBEs, we studied the $\mathrm{Ca}^{2+} / \mathrm{NLRP} 3 /$ caspase-1/GSDMD pathway. We found that CSE induced significant NLRP3 inflammasome activation and pyroptosis in 16HBE cells, as demonstrated by elevated protein levels of NLRP3 (Figures 4F,H), Cleavage Caspase-1 (Figures 4F,J), and GSDMD-N (Figures 4G,L), and decreased levels of Pro-caspase-1 (Figures 4F,I) and total GSDMD (Figures $\mathbf{4 G}, \mathbf{K}$ ). Interestingly, upon using pharmacological inhibition with GSK205, we observed attenuated NLRP3 inflammasome activation and pyroptosis induced by CSE, as demonstrated by decreased protein levels of NLRP3 (Figures 4F,H), Cleavage Caspase-1 (Figures 4F,J), and GSDMD-N (Figures 4G,L), and increased levels of Procaspase-1 (Figures 4F,I) and total GSDMD (Figures 4G,K). These results were confirmed by in vitro TRPV4 siRNA experiments. CSE-exposed TRPV4 siRNA 16HBEs showed decreased protein levels of NLRP3, Cleavage Caspase-1 and GSDMD-N, but increased levels of Pro-caspase-1 and total GSDMD compared with CSE-exposed wild type 16HBEs (Supplementary Figures 3F-L). As LDH release was also a marker of pyroptosis (Wu et al., 2018), we detected LDH release in supernants of $16 \mathrm{HBEs}$. Our results showed that, while CSE promoted the release of $\mathrm{LDH}$, pharmacological inhibition with GSK205 or TRPV4 gene knockdown attenuated CSE-induced $\mathrm{LDH}$ production (Figure $4 \mathrm{E}$ and Supplementary Figure $3 \mathrm{E}$ ). Moreover, we found that CSE could markedly increased PIpositive cells, which could be rescued by pharmacological inhibition with GSK205 (Figures 4M-P) or TRPV4 gene knockdown (Supplementary Figures 3M-Q). Taken together, these results indicate that TRPV4 mediates CSE-induced pyroptosis via the $\mathrm{Ca}^{2+}$ NLRP3/Caspase1/GSDMD pathway.

\section{Transient Receptor Potential Cation Channel Subfamily V Member Mediates CS Extract-Induced Inflammatory Gene Upregulation and Anti-Oxidant Gene Downregulation}

As CS-induced injury to AECs triggers production and releasing of cytokines, which lead to recruitment of macrophages and neutrophils, participating in the development and progression of COPD (Brusselle et al., 2011; Tuder and Petrache, 2012; Barnes, 2013), we explored whether TRPV4 was possibly involved in this process. Our results showed that pharmacological inhibition of TRPV4 with GSK205 abrogated CSE-induced gene expressions of $I L-8, I L-1 \beta$, and $I L-18$ (Figures 5A-C) while 


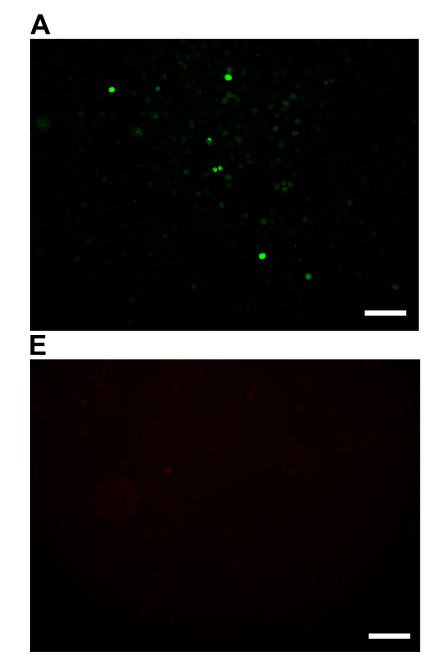

Control
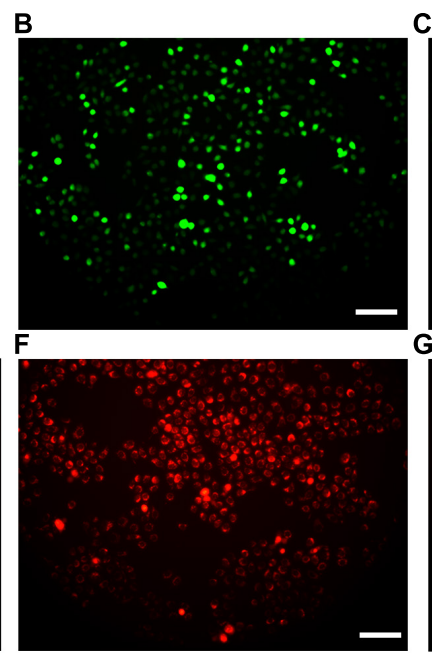

$4 \% \mathrm{CSE}$

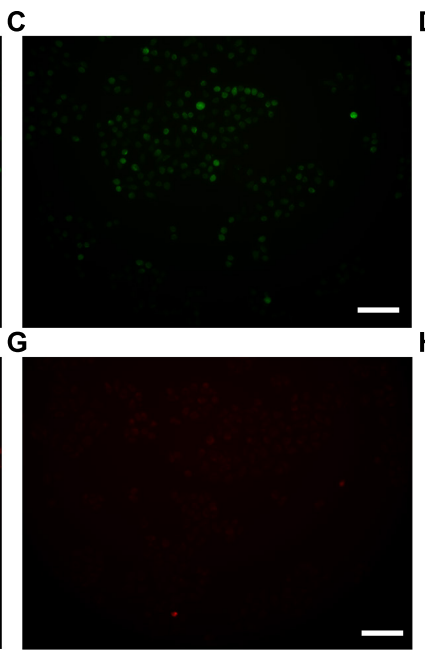

$4 \% \mathrm{CSE}+\mathrm{GSK} 10 \mathrm{uM}$

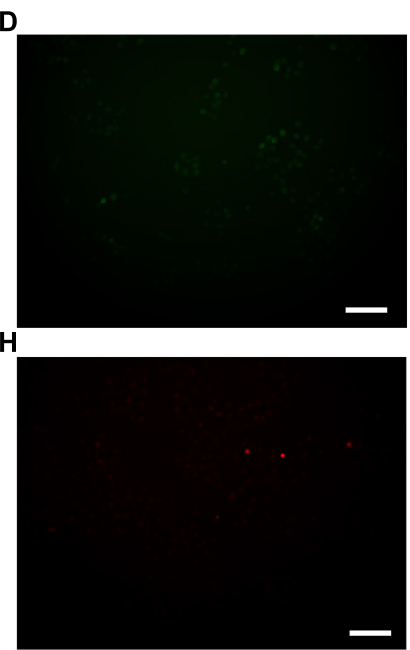

GSK $10 \mathrm{uM}$
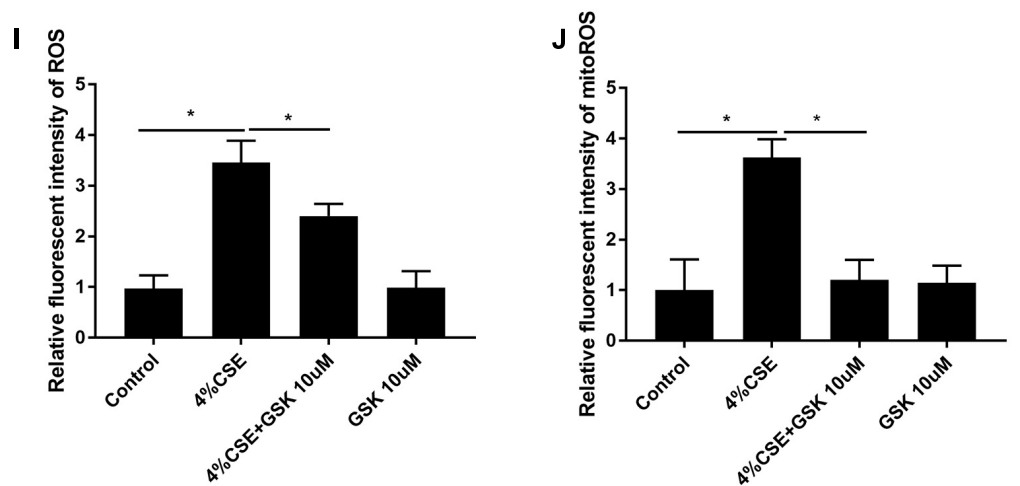

FIGURE 6 | Pharmacological inhibition of TRPV4 channel with GSK205 abrogated increased intracellular and mitochondrial ROS induced by CSE. 16HBEs were treated with GSK205 $(10 \mu \mathrm{M})$ for $1 \mathrm{~h}$, then stimulated with 4\% CSE for another $24 \mathrm{~h}$. (A-H) Representative photomicrographs of intracellular and mitochondrial ROS. Increased intracellular and mitochondrial ROS induced by CSE were attenuated pharmacological inhibition with GSK205. Bar: 100 $\mu \mathrm{m}$. (I,J) Quantification of photomicrographs from (A-H) using ImageJ software. ${ }^{*} P<0.05 . N=5$ independent experiments.

attenuated the downregulation of anti-oxidant genes NQO1, $M N S O D$, and CAT induced by CSE (Figures 5D-F). These findings were confirmed in CSE-exposed TRPV4 siRNA 16HBEs, by showing decreased $I L-8, I L-1 \beta$, and $I L-18$ gene expressions but increased NQO1, MNSOD, and CAT gene expressions, compared with CSE-exposed wild type 16HBEs (Supplementary Figures 6A-F). Taken together, these data demonstrate that TRPV4 mediates CSE-induced inflammatory gene upregulation and anti-oxidant gene downregulation.

\section{Transient Receptor Potential Cation Channel Subfamily V Member 4 Mediates CS Extract-Induced Intracellular and Mitochondrial Reactive Oxygen Species}

As ROS and mitochondrial ROS are involved in the assembly of NLRP3 (Abderrazak et al., 2015; Zhang et al., 2021), we thus measured the intracellular and mitochondrial ROS. Exposure to CSE for $24 \mathrm{~h}$ significantly elevated intracellular
ROS in 16HBEs, while pharmacological inhibition with GSK205 attenuated the effect of CSE (Figures 6A-D,I). Similar results were observed for the mitochondrial ROS level in these cells (Figures 6E-H,J). Additionally, this observation was further confirmed by TRPV4 siRNA experiments with 16HBEs (Supplementary Figures 7A-L).

\section{Transient Receptor Potential Cation Channel Subfamily V Member 4 Mediates CS Extract-Induced Damage of Mitochondrial Fitness}

Mitochondrial dysfunction is known to drive the inflammation associated with COPD (Wiegman et al., 2015). We detected the mitochondrial morphology and activity of 16HBEs after stimulation with CSE. For the detection of mitochondrial morphology, mitochondria were labeled with MitoTracker Green. The mitochondria in CSE-stimulated cells showed decreased relative fluorescence intensity, which could be rescued 

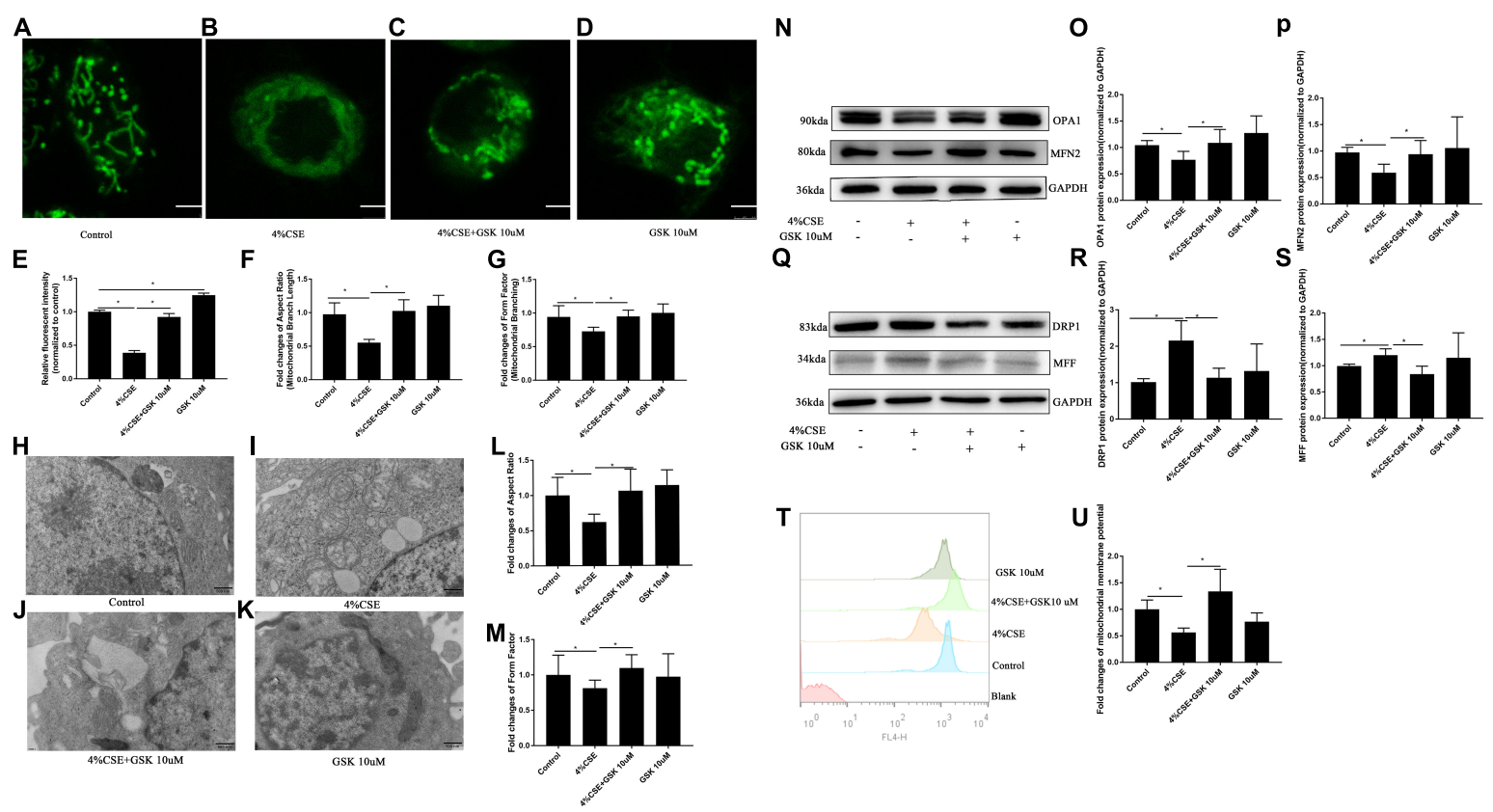

FIGURE 7 | Pharmacological inhibition of TRPV4 channel with GSK205 rescued mitochondrial damage induced by CSE. 16 HBEs were treated with GSK205 (10 $\mu \mathrm{M})$ for $1 \mathrm{~h}$, then stimulated with 4\% CSE for another $24 \mathrm{~h}$. (A-D) Representative images of mitochondrial morphology detected by the MitoTracker Green. Bar: 2.5 $\mu \mathrm{m}$. (E-G) Quantification of Mitotracker Green intensity, Aspect Ratio and Form Factor. Decreased MitoTracke Green intensity, Aspect Ratio and Form Factor induced by CSE were rescued by pharmacological inhibition with GSK205. * $P<0.05$. (H-K) Representative images of mitochondrial morphology detected by TEM. Bar: 500 nm. (L,M) Quantification of Aspect Ratio and Form Factor. Decreased Aspect Ratio and Form Factor induced by CSE were rescued by pharmacological inhibition with GSK205. ${ }^{*} P<0.05$. (N-P) Pharmacological inhibition with GSK205 rescued decreased protein level of OPA1 and MFN2 induced by CSE, (Q-S) while mitigated increased protein level of DRP1 and MFF induced by CSE. ${ }^{\star} P<0.05 . N \geq 5$ independent experiments. (T,U) Pharmacological inhibition with GSK205 rescued decreased MMP induced by CSE. ${ }^{*} P<0.05 . N \geq 3$.

by pharmacological inhibition with GSK205 (Figures 7AE) or by TRPV4 siRNA (Supplementary Figures 8A-F). Moreover, mitochondrial morphology was quantified using Aspect Ratio (length, parameter of mitochondrial fusion) and Form Factor (degree of branching, parameter of mitochondrial networking). The result demonstrated that CSE exposure also caused significant reduction of Aspect Ratio and Form Factor, which could be rescued by pharmacological inhibition with GSK205 (Figures 7F,G), or similarly by TRPV4 siRNA (Supplementary Figures $\mathbf{8 G}, \mathbf{H}$ ). Furthermore, ultrastructural analyses by transmission electron microscopy (TEM) observed a significant accumulation of damaged mitochondria with swollen and disrupted cristae in CSE-stimulated cells, which was mitigated by pharmacological inhibition with GSK205 (Figures $\mathbf{7 H}-\mathbf{K}$ ), as quantified by Aspect Ratio and Form Factor, parameters that reflect the complexity of mitochondrial morphology (Figures 7L,M). Likewise, TRPV4 siRNA 16HBEs showed alleviated mitochondrial damage compared to wild type 16HBEs (Supplementary Figures 8I-O). For the detection of mitochondrial activity, Western blotting analysis was carried out, which showed that CSE exposure for $24 \mathrm{~h}$ suppressed the expression of mitochondrial fusion protein OPA1 and MFN2, while pharmacological inhibition with GSK205 attenuated this suppression (Figures $7 \mathbf{N}-\mathbf{P}$ ). Moreover, exposure to CSE for $24 \mathrm{~h}$ enhanced the expression of mitochondrial fission proteins DRP1 and MFF, which could be abrogated by pharmacological inhibition with GSK205 (Figures 7Q-S). Compared to CSE-exposed wild type 16HBEs, TRPV4 siRNA 16HBEs showed increased protein levels of OPA1 and MFN2 while decreased DRP1 and MFF (Supplementary Figures 8PU). Additionally, flow cytometry analysis showed decreased MMP after CSE stimulation, while pharmacological inhibition with GSK205 (Figures 7T,U) or TRPV4 siRNA (Supplementary Figures $\mathbf{8 V}, \mathbf{W}$ ) attenuated this decline. In summary, these data indicate that TRPV4 mediates CSE-induced damage of mitochondrial fitness.

\section{DISCUSSION}

In the present study, for the first time to our knowledge, we observed heightened pyroptosis of AECs in both human and mouse COPD. At the same time, we also confirmed upregulation of TRPV4 in these patients and the animal model. More importantly, we found that TRPV4 mediated CSEinduced pyroptosis via the $\mathrm{Ca}^{2+} / \mathrm{NLRP} 3 / \mathrm{Caspase}-1 / \mathrm{GSDMD}$ axis, revealing a novel mechanism potentially involved in the pathogenesis of COPD.

Pyroptosis is a newly discovered form of proinflammatory cell death, defined as gasdermin-mediated programmed necrosis (Man et al., 2017; Manthiram et al., 2017; Swanson et al., 2019). Previous studies indicated that Caspase-1/4/5/11 could induce 


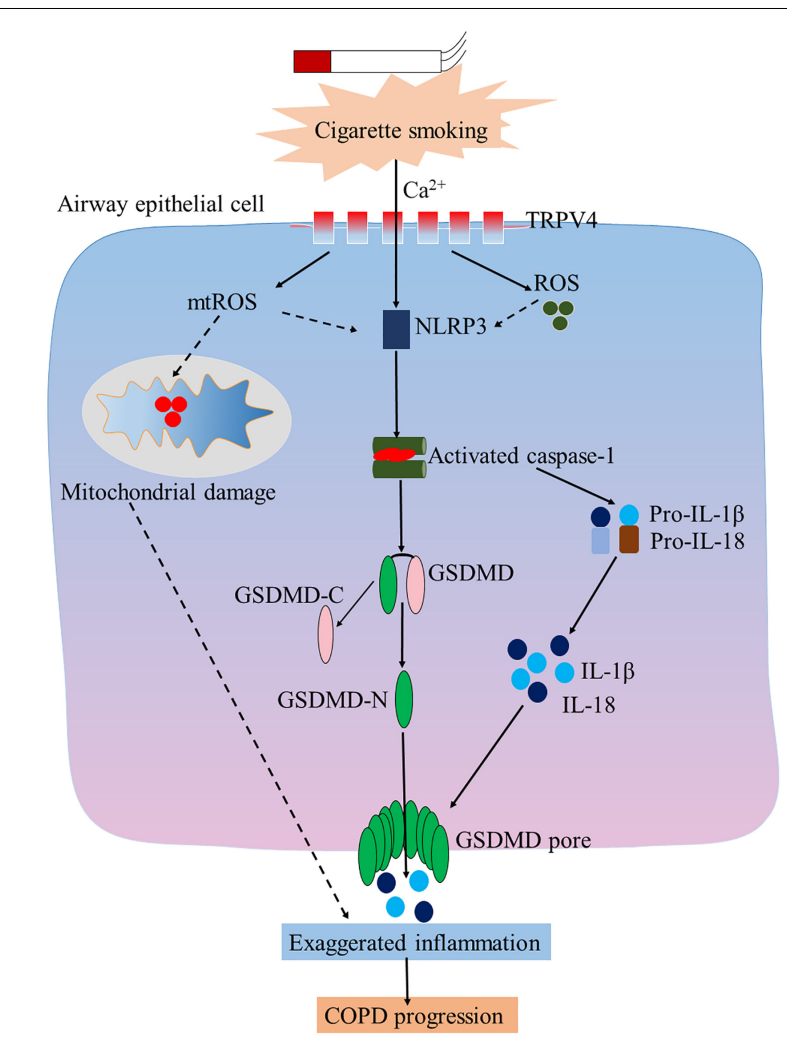

FIGURE 8 | TRP4 mediates CSE-induced pyroptosis airway epithelial cells. Inhibition of TRPV4 could alleviate CSE-induced inflammation, oxidative stress, and mitochondrial damage in airway epithelial cells.

pyroptosis via cleaving gasdermin D (GSDMD) (Kayagaki et al., 2015; Shi et al., 2015). During cleavage, the inhibitory GSDMD$\mathrm{C}$ domain was removed while the pore-forming GSDMD-N domain was unleashed for lysing the membranes (Aglietti et al., 2016; Ding et al., 2016; Liu et al., 2016; Sborgi et al., 2016). Although an in vitro study has implicated pyroptosis in the pathogenesis of COPD (Zhang et al., 2021), the expressions of pyroptosis in human and mouse COPD were not clear. In the current study, we found increased expressions of GSDMDN/GSDMD-C in AECs from both patients with COPD and a wellestablished mouse model of COPD. Additionally, our in vitro experiments demonstrated that CSE induced upregulation of GSDMD-N in 16 HBEs.

Up till now, the molecular pathways regulating pyroptosis in COPD remain to be investigated. The transient receptor potential (TRP) family is a large family of ion channel proteins which are subdivided into six groups: TRPV, TRPM, TRPA, TRPML, TRPP, and TRPC (Clapham, 2003; Negri et al., 2019). Accumulating evidence indicates that TRPV4 is involved in a variety of lung diseases, including cough (Bonvini et al., 2016; Bonvini and Belvisi, 2017), asthma (Yao et al., 2019), COPD (Baxter et al., 2014), idiopathic pulmonary fibrosis (Riteau et al., 2010), and acute respiratory distress syndrome (Balakrishna et al., 2014). However, the roles of TRPV4 in lung diseases are complex, since both protective and deleterious actions have been reported, depending on the models under study (Scheraga et al., 2020). Interestingly, a previous study indicated that TRPV4 was involved in the pathogenesis of COPD, and CSE-induced ATP and IL-1 $\beta$ release in vitro and in vivo (mouse model) was mediated by TRPV4 (Baxter et al., 2014), indicating that TRPV4 may be a potential therapeutic target for COPD. Emerging data supported that $\mathrm{Ca}^{2+}$ influx is critical for pyroptosis (Wang et al., 2020), however, the mechanisms by which $\mathrm{Ca}^{2+}$ influx promotes pyroptosis via the TRPV4 channel have not been elucidated. Thus, we asked whether TRPV4 mediated pyroptosis of AECs induced by CS exposure. In the current study, we demonstrated that CSE stimulation promoted $\mathrm{Ca}^{2+}$ influx, increased the expression of NLRP3, caspase-1, GSDMD-N, and increased the number of $\mathrm{PI}^{+}$cells, which are consistent with previous reports (Wang et al., 2019; Yoo et al., 2020). Interestingly, we found that pharmacological inhibition with GSK205 or knockdown of TRPV4 function attenuated CSE-induced pyroptosis through the $\mathrm{Ca}^{2+} / \mathrm{NLRP} 3 /$ caspase1/GSDMD pathway, as shown by decreased NLRP3, caspase1, GSDMD-N expression, and decreased $\mathrm{PI}^{+}$cell numbers, indicating that TRPV4 is critical for CS-induced pyroptosis. In addition, we observed increased IL-1 $\beta$, IL18, and LDH release in $16 \mathrm{HBE}$ exposed to CSE, which was in line with the consequence of enhanced pyroptosis. In fact, previous studies had reported upregulation of IL- $1 \beta$ and IL-18 in bronchoalveolar lavage from mouse models of COPD (Rovina et al., 2009; Sapey et al., 2009) as well as from COPD patients (Lappalainen et al., 2005; Lucattelli et al., 2011).

It is well-known that $\mathrm{Ca}^{2+}$ signaling is also critical for ROS and mitochondrial ROS (Jiang et al., 2011; Güzel et al., 2021), which are involved in the assembly of NLRP3 (Abderrazak et al., 2015; Zhang et al., 2021). In the current study, we observed that CSE induced ROS and mitochondrial ROS production via TRPV4 channel. Emerging evidence indicates that dysfunctional mitochondria enhanced inflammation in COPD (Wiegman et al., 2015). In our study, we observed an imbalance of mitochondrial fission and fusion proteins induced by CSE, while pharmacological inhibition with GSK205 or knockdown of TRPV4 function may be capable of preventing this abnormality, providing further evidence supporting TRPV4 as a potential candidate of therapy for CS-associated COPD.

One of the limitations of our study is that, although we demonstrated that TRPV4 mediated CSE-induced pyroptosis via the $\mathrm{Ca}^{2+} / \mathrm{NLRP} 3 / \mathrm{Caspase}-1 / \mathrm{GSDMD}$ axis in vitro experiments, the significance of pyroptosis and this signaling pathway in COPD pathogenesis still needs further investigation in mouse models and human COPD.

\section{CONCLUSION}

In conclusion, as shown in the proposed schematic model (Figure 8), our study reveals a novel role of TRPV4 in mediating pyroptosis of AECs from COPD. TRPV4 mediated CSE-induced pyroptosis via the $\mathrm{Ca}^{2+}$ / NLRP3/Caspase-1/GSDMD axis, suggesting a potential mechanism involved in the pathogenesis of COPD. TRPV4 
also mediated mitochondrial damage induced by CSE. These results provide evidence for a new pathway in the pathogenic mechanisms of the disease, and hopefully for exploring interventional target for therapy of COPD.

\section{DATA AVAILABILITY STATEMENT}

The raw data supporting the conclusions of this article will be made available by the authors, without undue reservation.

\section{ETHICS STATEMENT}

The studies involving human participants were reviewed and approved by the Ethics Review Committee of Peking University Third Hospital. The patients/participants provided their written informed consent to participate in this study. The animal study was reviewed and approved by the Animal Care Committee of Peking University Third Hospital.

\section{REFERENCES}

Abderrazak, A., Syrovets, T., Couchie, D., El Hadri, K., Friguet, B., Simmet, T., et al. (2015). NLRP3 inflammasome: from a danger signal sensor to a regulatory node of oxidative stress and inflammatory diseases. Redox Biol. 4, 296-307. doi: 10.1016/j.redox.2015.01.008

Aglietti, R. A., Estevez, A., Gupta, A., Ramirez, M. G., Liu, P. S., Kayagaki, N., et al. (2016). GsdmD p30 elicited by caspase-11 during pyroptosis forms pores in membranes. Proc. Natl. Acad. Sci. U.S.A. 113, 7858-7863. doi: 10.1073/pnas. 1607769113

Balakrishna, S., Song, W., Achanta, S., Doran, S. F., Liu, B., Kaelberer, M. M., et al. (2014). TRPV4 inhibition counteracts edema and inflammation and improves pulmonary function and oxygen saturation in chemically induced acute lung injury. Am. J. Physiol. Lung Cell. Mol. Physiol. 307, L158-L172. doi: 10.1152/ ajplung.00065.2014

Barnes, P. J. (2013). New anti-inflammatory targets for chronic obstructive pulmonary disease. Nat. Rev. Drug Discov. 12, 543-559. doi: 10.1038/nrd 4025

Baxter, M., Eltom, S., Dekkak, B., Yew-Booth, L., Dubuis, E. D., Maher, S. A., et al. (2014). Role of transient receptor potential and pannexin channels in cigarette smoke-triggered ATP release in the lung. Thorax 69, 1080-1089. doi: 10.1136/thoraxjnl-2014-205467

Bonvini, S. J., and Belvisi, M. G. (2017). Cough and airway disease: the role of ion channels. Pulm. Pharmacol. Ther. 47, 21-28. doi: 10.1016/j.pupt.2017.06.009

Bonvini, S. J., Birrell, M. A., Grace, M. S., Maher, S. A., Adcock, J. J., Wortley, M. A., et al. (2016). Transient receptor potential cation channel, subfamily V, member 4 and airway sensory afferent activation: role of adenosine triphosphate. J. Allerg. Clin. Immunol. 138, 249-261.e12. doi: 10.1016/j.jaci.2015.10.044

Broz, P., and Dixit, V. M. (2016). Inflammasomes: mechanism of assembly, regulation and signalling. Nat. Rev. Immunol. 16, 407-420. doi: 10.1038/nri. 2016.58

Brusselle, G. G., Joos, G. F., and Bracke, K. R. (2011). New insights into the immunology of chronic obstructive pulmonary disease. Lancet 378, 1015-1026. doi: 10.1016/s01406736(11)60988-4

Clapham, D. E. (2003). TRP channels as cellular sensors. Nature 426, 517-524. doi: 10.1038/nature02196

Ding, J., Wang, K., Liu, W., She, Y., Sun, Q., Shi, J., et al. (2016). Pore-forming activity and structural autoinhibition of the gasdermin family. Nature 535, 111-116. doi: 10.1038/nature18590

Feng, Y. S., Tan, Z. X., Wu, L. Y., Dong, F., and Zhang, F. (2020). The involvement of NLRP3 inflammasome in the treatment of Alzheimer's disease. Ageing Res. Rev. 64:101192. doi: 10.1016/j.arr.2020.101192

\section{AUTHOR CONTRIBUTIONS}

YR and YS designed the experiments and wrote the manuscript. YR, XG, JX, and YL performed the experiments. All authors contributed to the article and approved the submitted version.

\section{FUNDING}

This study was financially supported by the National Natural Science Foundation of China (81970041, $81770040)$ and the Natural Science Foundation of Beijing Municipality (7192224).

\section{SUPPLEMENTARY MATERIAL}

The Supplementary Material for this article can be found online at: https://www.frontiersin.org/articles/10.3389/fphys. 2021.783891/full\#supplementary-material

Gao, L., Dong, X., Gong, W., Huang, W., Xue, J., Zhu, Q., et al. (2021). Acinar cell NLRP3 inflammasome and GSDMD activation mediates pyroptosis and systemic inflammation in acute pancreatitis. Br. J. Pharmacol. 178, 3533-3552. doi: 10.1111/bph.15499

Güzel, M., Nazı roðlu, M., Akpı nar, O., and Çı nar, R. (2021). Interferon Gammamediated oxidative stress induces apoptosis, neuroinflammation, zinc ion influx, and TRPM2 channel activation in neuronal cell line: modulator role of curcumin. Inflammation 44, 1878-1894. doi: 10.1007/s10753-021-01465-4

Jiang, F., Zhang, Y., and Dusting, G. J. (2011). NADPH oxidase-mediated redox signaling: roles in cellular stress response, stress tolerance, and tissue repair. Pharmacol. Rev. 63, 218-242. doi: 10.1124/pr.110.002980

Jorgensen, I., and Miao, E. A. (2015). Pyroptotic cell death defends against intracellular pathogens. Immunol. Rev. 265, 130-142. doi: 10.1111/imr. 12287

Kayagaki, N., Stowe, I. B., Lee, B. L., O’Rourke, K., Anderson, K., Warming, S., et al. (2015). Caspase-11 cleaves gasdermin D for non-canonical inflammasome signalling. Nature 526, 666-671. doi: 10.1038/nature15541

Lappalainen, U., Whitsett, J. A., Wert, S. E., Tichelaar, J. W., and Bry, K. (2005). Interleukin-1beta causes pulmonary inflammation, emphysema, and airway remodeling in the adult murine lung. Am. J. Respir. Cell Mol. Biol. 32, 311-318. doi: 10.1165/rcmb.2004-0309OC

Liu, X., Zhang, Z., Ruan, J., Pan, Y., Magupalli, V. G., Wu, H., et al. (2016). Inflammasomeactivated gasdermin $\mathrm{D}$ causes pyroptosis by forming membrane pores. Nature 535, 153-158. doi: 10.1038/nature18629

Lucattelli, M., Cicko, S., Müller, T., Lommatzsch, M., De Cunto, G., Cardini, S., et al. (2011). P2X7 receptor signaling in the pathogenesis of smoke-induced lung inflammation and emphysema. Am. J. Respir. Cell. Mol. Biol. 44, 423-429. doi: $10.1165 / \mathrm{rcmb} .2010-0038 \mathrm{OC}$

Man, S. M., Karki, R., and Kanneganti, T. D. (2017). Molecular mechanisms and functions of pyroptosis, inflammatory caspases and inflammasomes in infectious diseases. Immunol. Rev. 277, 61-75. doi: 10.1111/imr.12534

Manthiram, K., Zhou, Q., Aksentijevich, I., and Kastner, D. L. (2017). The monogenic autoinflammatory diseases define new pathways in human innate immunity and inflammation. Nat. Immunol. 18, 832-842. doi: 10.1038/ni.3777

Negri, S., Faris, P., Berra-Romani, R., Guerra, G., and Moccia, F. (2019) Endothelial transient receptor potential channels and vascular remodeling: extracellular $\mathrm{Ca}(2+)$ entry for angiogenesis, arteriogenesis and vasculogenesis. Front. Physiol. 10:1618. doi: 10.3389/fphys.2019.01618

Rathinam, V. A. K., Zhao, Y., and Shao, F. (2019). Innate immunity to intracellular LPS. Nat. Immunol. 20, 527-533. doi: 10.1038/s41590-019-0368-3

Riteau, N., Gasse, P., Fauconnier, L., Gombault, A., Couegnat, M., Fick, L., et al. (2010). Extracellular ATP is a danger signal activating P2X7 receptor in lung 
inflammation and fibrosis. Am. J. Respir. Crit. Care Med. 182, 774-783. doi: 10.1164/rccm.201003-0359OC

Rovina, N., Dima, E., Gerassimou, C., Kollintza, A., Gratziou, C., and Roussos, C. (2009). Interleukin-18 in induced sputum: association with lung function in chronic obstructive pulmonary disease. Respir. Med. 103, 1056-1062. doi: 10.1016/j.rmed.2009.01.011

Sapey, E., Ahmad, A., Bayley, D., Newbold, P., Snell, N., Rugman, P., et al. (2009). Imbalances between interleukin-1 and tumor necrosis factor agonists and antagonists in stable COPD. J. Clin. Immunol. 29, 508-516. doi: 10.1007/ s10875-009-9286-8

Sborgi, L., Rühl, S., Mulvihill, E., Pipercevic, J., Heilig, R., Stahlberg, H., et al. (2016). GSDMD membrane pore formation constitutes the mechanism of pyroptotic cell death. EMBO J. 35, 1766-1778. doi: 10.15252/embj.201694696

Scheraga, R. G., Southern, B. D., Grove, L. M., and Olman, M. A. (2020). The role of TRPV4 in regulating innate immune cell function in lung inflammation. Front. Immunol. 11:1211. doi: 10.3389/fimmu.2020.01211

Shi, J., Zhao, Y., Wang, K., Shi, X., Wang, Y., Huang, H., et al. (2015). Cleavage of GSDMD by inflammatory caspases determines pyroptotic cell death. Nature 526, 660-665. doi: 10.1038/nature15514

Singh, D., Agusti, A., Anzueto, A., Barnes, P. J., Bourbeau, J., Celli, B. R., et al. (2019). Global strategy for the diagnosis, management, and prevention of chronic obstructive lung disease: the GOLD science committee report 2019. Eur. Respir. J. 53:1900164. doi: 10.1183/13993003.00164-2019

Swanson, K. V., Deng, M., and Ting, J. P. (2019). The NLRP3 inflammasome: molecular activation and regulation to therapeutics. Nat. Rev. Immunol. 19, 477-489. doi: 10.1038/s41577-0190165-0

Tuder, R. M., and Petrache, I. (2012). Pathogenesis of chronic obstructive pulmonary disease. J. Clin. Invest. 122, 2749-2755. doi: 10.1172/jci60324

Wang, D., Zheng, J., Hu, Q., Zhao, C., Chen, Q., Shi, P., et al. (2020). Magnesium protects against sepsis by blocking gasdermin D N-terminalinduced pyroptosis. Cell Death Diff. 27, 466-481. doi: 10.1038/s41418-0190366-x

Wang, X., Bian, Y., Zhang, R., Liu, X., Ni, L., Ma, B., et al. (2019). Melatonin alleviates cigarette smoke-induced endothelial cell pyroptosis through inhibiting ROS/NLRP3 axis. Biochem. Biophys. Res. Commun. 519, 402-408. doi: 10.1016/j.bbrc.2019.09.005

Wiegman, C. H., Michaeloudes, C., Haji, G., Narang, P., Clarke, C. J., Russell, K. E., et al. (2015). Oxidative stress-induced mitochondrial dysfunction drives inflammation and airway smooth muscle remodeling in patients with chronic obstructive pulmonary disease. J. Allerg. Clin. Immunol. 136, 769-780. doi: 10.1016/j.jaci.2015.01.046

Wu, X., Zhang, H., Qi, W., Zhang, Y., Li, J., Li, Z., et al. (2018). Nicotine promotes atherosclerosis via ROS-NLRP3-mediated endothelial cell pyroptosis. Cell Death Dis. 9:171. doi: 10.1038/s41419-017$0257-3$
Xiao, C., Zhao, H., Zhu, H., Zhang, Y., Su, Q., Zhao, F., et al. (2020). Tisp40 Induces tubular 481 epithelial cell GSDMD-mediated pyroptosis in renal Ischemiareperfusion injury via NF $\kappa B$ signaling. Front. Physiol. 11:906. doi: 10.3389/ fphys.2020.00906

Xie, Y., Huang, Y., Ling, X., Qin, H., Wang, M., and Luo, B. (2020). Chemerin/CMKLR1 Axis 484 promotes inflammation and pyroptosis by activating NLRP3 inflammasome in diabetic cardiomyopathy rat. Front. Physiol. 11:381. doi: 10.3389/fphys.2020.0 0381

Xiong, J., Zhou, L., Tian, J., Yang, X., Li, Y., Jin, R., et al. (2020). Cigarette smoke-induced 487 lymphoid neogenesis in COPD involves IL17/RANKL pathway. Front. Immunol. 1:588522. doi: 10.3389/fimmu.2020.58 8522

Yao, L., Chen, S., Tang, H., Huang, P., Wei, S., Liang, Z., et al. (2019). Transient receptor potentia ion channels mediate adherens junctions dysfunction in a toluene diisocyanate-induced murine asthma model. Toxicol. Sci. 168, 160-170. doi: $10.1093 /$ toxsci/kfy 285

Yoo, Y. M., Jung, E. M., Jeon, B. H., Tran, D. N., and Jeung, E. B. (2020). Cigarette smoke extraxt influences intracellular calcium concentration in A549 cells. J. Physiol. Pharmacol. 71:132. doi: 10.26402/jpp. 2020.5.08

Zhang, M. Y., Jiang, Y. X., Yang, Y. C., Liu, J. Y., Huo, C., Ji, X. L., et al. (2021). Cigarette smoke extract induces pyroptosis in human bronchial epithelial cells through the ROS/NLRP3/caspase-1 pathway. Life Sci. 269:119090. doi: 10.1016/ j.lfs. 2021.119090

Zhou, L., Le, Y., Tian, J., Yang, X., Jin, R., Gai, X., et al. (2019). Cigarette smoke-induced RANKL expression enhances MMP-9 production by alveolar macrophages. Int. J. Chron. Obstruct. Pulmon. Dis. 14, 81-91.

Conflict of Interest: The authors declare that the research was conducted in the absence of any commercial or financial relationships that could be construed as a potential conflict of interest.

Publisher's Note: All claims expressed in this article are solely those of the authors and do not necessarily represent those of their affiliated organizations, or those of the publisher, the editors and the reviewers. Any product that may be evaluated in this article, or claim that may be made by its manufacturer, is not guaranteed or endorsed by the publisher.

Copyright (ㄷ 2021 Rao, Gai, Xiong, Le and Sun. This is an open-access article distributed under the terms of the Creative Commons Attribution License (CC BY). The use, distribution or reproduction in other forums is permitted, provided the original author(s) and the copyright owner(s) are credited and that the original publication in this journal is cited, in accordance with accepted academic practice. No use, distribution or reproduction is permitted which does not comply with these terms. 